\title{
A computer-controlled matrix system for presentation to the skin of complex spatiotemporal patterns
}

\author{
ROGER W. CHOLEWIAK and CARL E. SHERRICK \\ Princeton University, Princeton, New Jersey 08544
}

\begin{abstract}
A system for presenting complex spatiotemporal patterns to the skin is described. The computer-controlled device consists of a matrix of 64 piezoceramic benders mounted in a support system that can be fitted to steeply contoured skin surfaces such as the thigh. Vibrotactile patterns that include movement across the skin's surface and/or intensitive variation within or across display epochs are programmed and presented in a frame-by-frame manner, similar to the production of motion pictures. Patterns can be predefined, or modified by observers' responses to allow for an interactive haptographic display. Design considerations for the construction of the tactile matrix, computer interface, and software are discussed.
\end{abstract}

Attempts to investigate the pattern-processing capabilities of the skin have employed a variety of devices that have ranged from the experimenter's fingertip ("graphesthesia"), stroking brushes (Dreyer, Hollins, \& Whitsel, 1978), and cookie cutters (Gibson, 1962) to more complex devices involving one or many individual punctate mechanical stimuli. Historically, the most useful of these has been the set of tactile patterns that make up the braille language for the blind. More recent attempts to elaborate on the matrix concept to produce a dynamic display have involved large numbers of contactors (often driven by solenoids) set into an array applied to some portion of the subject's skin (Bliss, 1970; Geldard, 1974). These have included the 20 by 20 array in the TVSS system at the Smith-Kettlewell Institute (Collins, 1970), the 10 by 10 array in the Kinotact (Craig, 1974), and the 6 by 24 element array used in the Optacon, a tactile aid for the blind (Bliss, 1974; Bliss, Katcher, Rogers, \& Shepard, 1970). Although such systems can provide a rich and varied display for the presentation of tactile patterns, a limiting factor in each is the method of controlling the patterns presented by the devices. Typically, the electromechanical array has been coupled, through the appropriate electronic circuitry, to a camera photographing the pattern to be presented. A spatiotemporal display involving pattern movement thus requires moving the camera or moving the patterns. Furthermore, owing to the manner of coupling of the camera to the display,

This work was supported by Grant NS 04755 from the National Institutes of Health to Princeton University. We would like to thank Paul Condit for writing the basic version of the matrix software, Thomas Temple for constructing the parallel interface and matrix controller, and Jerry Palin for coordinating the early phases of the system's development, as well as for designing the D/A converter and amplifier circuitry. minimal intensitive variation is possible, typically involving a binary (on or off) change.

Recently, several attempts have been made to circumvent these limitations through computer control of the arrays (Craig \& Sherrick, in press). We have developed such a system that provides for complete and independent control of the spatial, temporal, and intensitive parameters on each element of a large vibrotactile matrix. The basic design of the system allows the experimenter to duplicate the display of any existing device by the appropriate programming of the stimulus parameters. Thus the "Times Square" display of the Optacon, in which letters sweep across the skin's surface, can be presented, as well as nonmoving figures, slit-scanned displays, and patterns that involve changes in intensity either within the display epoch or across successive frames. Furthermore, patterns in which various dimensions of acoustic signals (such as speech) are spatiotemporally encoded can easily be presented. The device has been used in a variety of experiments to examine some of the more basic pattern-processing capabilities of the skin, such as the identification of patterns presented in different modes (Sherrick \& Cholewiak, Note 1), the effects of communality on tactile pattern discrimination (Gottheil, Cholewiak, \& Sherrick, 1978), and the relationship between numbers of active contactors and perceived intensity (Cholewiak, 1979).

\section{THE VIBROTACTILE MATRIX}

The vibrotactile matrix was designed to provide a relatively dense array of contactors capable of being fitted to rather steeply contoured skin surfaces. For example, a matrix that is approximately $11 \times 11 \mathrm{~cm}$ contacting the top surface of the thigh should be able to provide for a contactor-height disparity across its surface of approximately $2 \mathrm{~cm}$. Furthermore, the 
strength of the stimulus required for such a relatively insensitive surface demands stimulators that can produce mechanical displacements that range upward of 100 micrometers $(.1 \mathrm{~mm})$.

The electromechanical transducers chosen for the stimulators were $13 \times 32 \mathrm{~mm}$ piezoceramic reed benders (purchased from Gulton Industries, Metuchen, New Jersey). These devices bend when a voltage difference is applied across their faces, with the direction of movement of the free end being dependent on the polarity of the applied voltage. ${ }^{1}$ The Optacon uses similar benders, but they are smaller and are in a different configuration. The force and amplitude of the stimulus produced by the Optacon's 1 × $25 \mathrm{~mm}$ benders, although quite palpable by the fingertip, would be inadequate for most other body surfaces. This is due to the fact that while the displacement produced by the bender is a function of its length for a given input voltage and sinusoidal signal frequency, the force produced is a function of its width.

Sixty-four of the wider benders were vertically mounted in an 8 by 8 square array on $16-\mathrm{mm}(5 / 8$-in.) centers. In such a configuration, the applied voltage would produce a mechanical motion tangential to the skin's surface. For a given waveform (e.g., sinusoidal bursts, rectangular pulses, or haversine pulses), there exists no qualitative difference between tangential and perpendicular stimulation. (Changes in mechanical impedance on the skin with stimulus frequency have been reported to be a function of stimulus direction, however; Alles, 1970.) Contact with the skin was provided by attaching hemispherical nylon nuts to the edge of each bender with epoxy cement. A schematic drawing of a portion of the array and the mounting clamps is provided in Figure 1.

Each of the 64 benders is individually mounted with a $25-\mathrm{mm}$ free length in a $16 \times 16 \times 38 \mathrm{~mm}$ aluminum clamp that is, in turn, suspended in the array with a 60-mm-long smooth-shank screw. The shank rides in a hole in a glass epoxy board guide plate, allowing for relative vertical displacements of the contactor tips of approximately $30 \mathrm{~mm}$ when the array is in its freemoving state. This mounting arrangement also permits easy replacement of damaged benders, an infrequent occurrence despite their fragility. The box in which the clamping bars are supported is constructed of 6.4-mm polished aluminum plate stock. The electrical connections are made through a 68-terminal socket (Mini-Jones, TRW) mounted on the top of the matrix box, A standard ribbon cable connects the matrix to the computer driving system. The ground side of the electrical circuit for each element is the aluminum clamping bar, with individual voitage inputs provided to the opposite faces of the benders through an insulated plate within each clamping slot. In operation, the array is gently pressed onto the skin's surface, displacing the elements accordingly. The whole matrix is then clamped to hold the resulting contour by tightening a

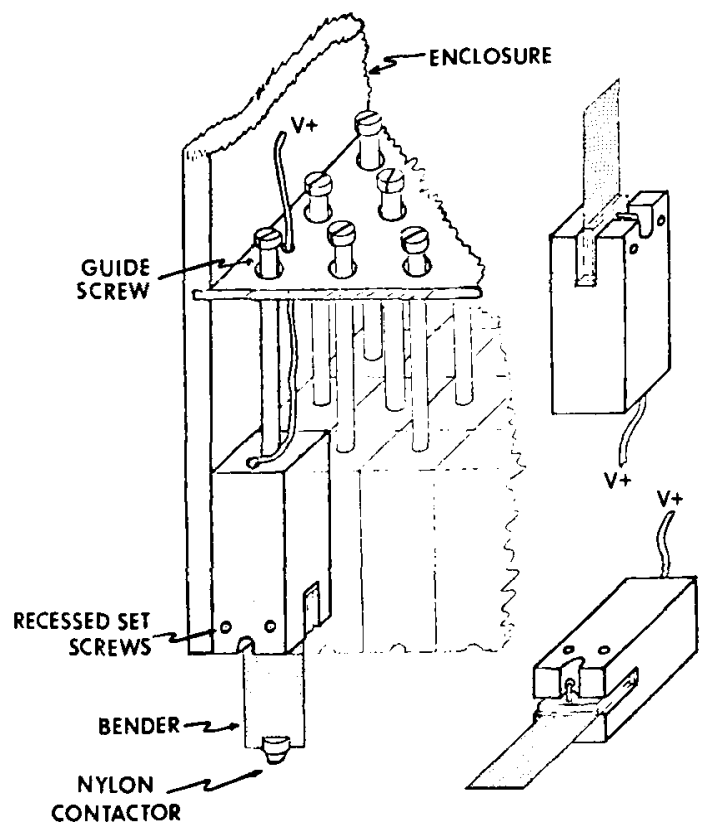

Figure 1. A schematic drawing of a portion of the vibrotactile matrix and the bender clamps. Signal ground is provided through the aluminum clamping blocks, with $\mathrm{V}+$ individually connected to each of the 64 elements. The guide screws allow for relative vertical displacements of the matrix elements, and tightening bolts (not shown) fix the array to the counter desired.

series of Delrin bolts placed in the side of the enclosure, thus compressing the bender clamp bars against each other. A weight is added to the fitted array to provide approximately $10 \mathrm{~g}$ of force per contactor. This arrangement provides for at least $20 \mathrm{~dB}$ of mechanical isolation between adjacent driving points, with most of the coupling taking place through the skin rather than through the matrix, due to its mass.

The amplitude of displacement produced for a given input voltage is monitored with an optically calibrated strain gauge that is cemented to the surface of the bender. Such devices are available through several vendors with various gauge factors (i.e., sensitivity). The most sensitive are semiconductor strain gauges (e.g., the Pixie, Endevco Corporation, or the semiconductor line from BLH Electronics). The system is calibrated by mounting the transducer on a bender (with epoxy or cyanoacrylic cement) and driving the bender with a sinusoidal signal. The changes in resistance resulting from the changes in the length of the strain gauge element as it undergoes compression and extension are monitored through a circuit, such as those shown in Figure 2, that converts the changes in resistance to a change in voltage.

The motion of the stimulator tip is visualized through a microscope with a filar micrometer eyepiece and stroboscopic illumination slightly offset from the frequency of the driving signal. The amplitude of displacement is then related to the magnitude of the output voltage from the monitor circuit. Once that 


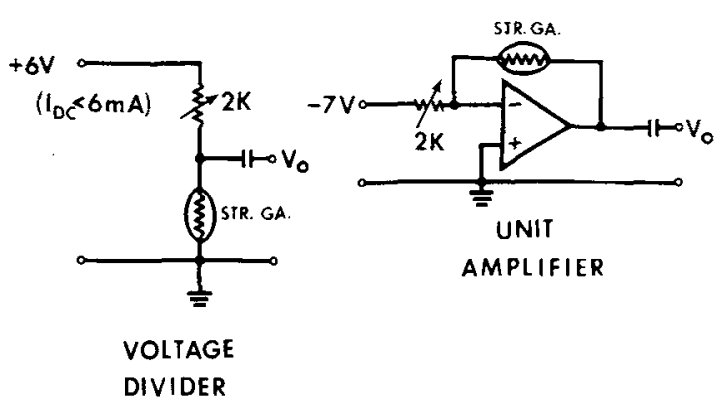

Figure 2. Two circuits used to monitor the change in resistance of semiconductor strain gauges. The $2 \mathrm{~K}$ variable resistors are used to tune the circuit for the particular gauge and, for the unit amplifier, the amplification required. The values for the capacitors depend on the application and frequency response desired.
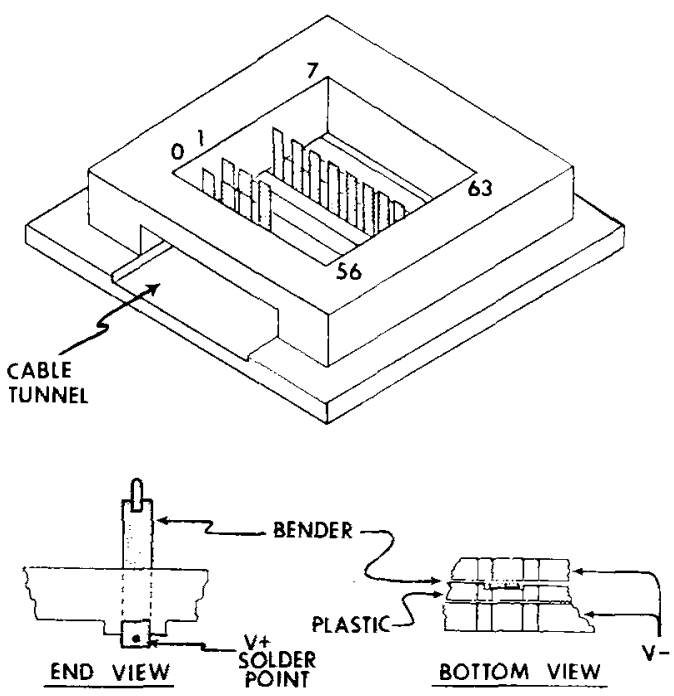

Figure 3. A schematic drawing of the small vibrotactile matrix designed to fit the palm of the hand, with several mounted benders. A contactor is shown on the bender in the end view. The $64 \mathrm{~V}+$ lines enter the array through the cable tunnel. Each $V+$ line is attached to its associated bender, and a single ground is common to all elements.

relationship is known, any damping or modification of the tip's motion during operation is reflected directly in the amplitude and waveform of the displacement monitor voltage, provided that the bender is flexing in its simplest mode. Only when static forces are quite large does other than this mode appear. Such a monitor could be incorporated into a feedback control system to provide for a constant-amplitude control of the system's output.

The $4.2-\mathrm{kg}$ array itself is supported by a counterweighted pulley system that allows the matrix to be lowered to or raised from the skin's surface while maintaining the appropriate contour for the area of skin to be stimulated. A similar matrix patterned after the one described here has been fabricated by the
Research Laboratory of Electronics at M.I.T. (Rubin, 1978), with a support arrangement consisting of a large counterbalanced lever, similarly allowing for ease in positioning.

A second, smaller, tactile matrix has also been constructed to fit the palm of the hand. It consists of 64 bender elements, each $6.4 \times 32 \mathrm{~mm}$ long, mounted at a fixed height in an upright position with $25 \mathrm{~mm}$ free length on approximately 8-mm centers (see Figure 3).

The fitting to the palm's contours was established by creating plaster casts of several hands, measuring the average depth of indentation for each point on the matrix, and permanently adjusting the contactor height for that depth. Typically, this array is mounted on the armrest of a modified dental chair, and the observer places his palm down on the contoured surface. Individual fitting for each observer involves presenting a pattern consisting of sequential taps on the individual loci. The hand, stabilized by a small sandbag, is then adjusted until the series of taps appears to produce a line that is continuous across the palm's surface. This method has proved quite satisfactory with dozens of different student observers. Even so, the present design is considered as a prototype, and a redesign of this matrix is anticipated to provide for the flexibility of adjustment incorporated into the larger matrix.

\section{THE COMPUTER INTERFACE}

The computer interface and driving circuitry were specifically designed for the PDP-8/f computer, which has a 12-bit word. The amplifiers were designed to optimize the response of the system at approximately $250 \mathrm{~Hz}$, the sinusoidal frequency to which the skin is generally most sensitive (Geldard, 1972). In addition, the typical driving waveform was intended to consist of single pulses of a $250-\mathrm{Hz}$ haversine. The overall design of the system includes the computer output board, which loads and enables a bank of 64 largely independent parallel signal control circuits, each of which is connected to an independent signal amplifier directly tied to one element of the tactile matrix. A block diagram of the entire system is shown in Figure 4.

The system provides for a frame-by-frame loading of all of the elements in the array with some intensity (ranging from 0 to $63 / 64$ of the maximum output voltage) and then pulses the driving system with the signal to be presented. It is only at this stage that channel communality exists, since only one signal input is provided to the entire array. After presentation of a particular spatiointensitive array, the signal may be repeated or another frame may be loaded. Thus, by specifying the sequential changes in frame rate, frame repetition, and active loci per frame, any spatiotemporal pattern can be generated.

Specifically, the system begins with the computer output board. The output board is simply a parallel interface in which the 12 bits of the computer word are 


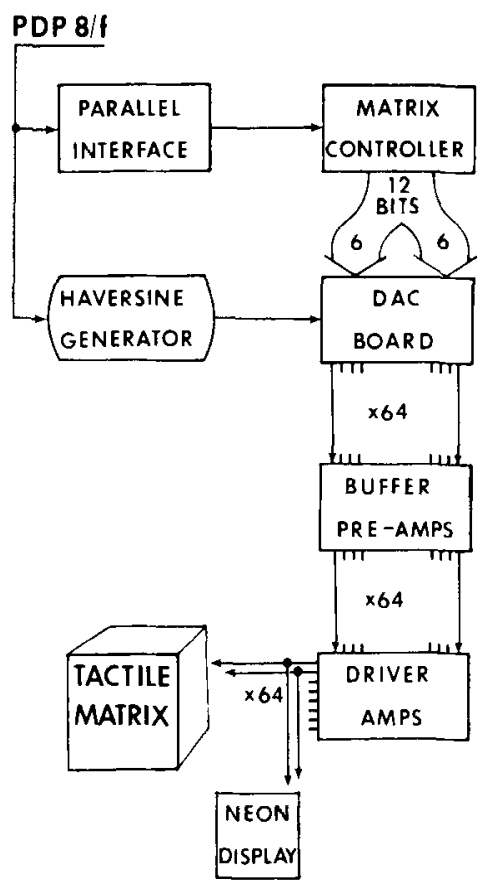

Figure 4. One of the 64 parallel channels in the tactile matrix control system, described in the text. The particular component values shown were chosen for a system tuned to approximately $250-\mathrm{Hz}$ haversines and for the electrical characteristics of the piezoceramic benders employed in the tactile matrix.

presented to the matrix control board. At this point, the word is divided into two 6-bit bytes (left and right 6 bits). Each byte contains the intensitive information for one of the matrix channels. If a byte specifies a zero level, its addressed element will not be activated when the frame is presented to the observer. The matrix control board serially loads 64 digital-to-analog (D/A) converters with the 6 bits of intensity information. This sequence is controlled by a simple multiplexer counting circuit that switches input words to successive pairs of D/A converters until all have been loaded. Loading the 64 channels takes approximately 320 microsec with the
PDP-8/f. In this particular configuration, the D/A converters act essentially as digital attenuators, set to some level, waiting for the signal they are to control. When all the channels have been loaded, the V-ref input of the $\mathrm{D} / \mathrm{A}$ converters are presented with the stimulus waveform by appropriately triggering a haversine generator or gating a function generator for some specified amount of time. The input signal is appropriately attenuated by each channel's D/A converter and ranges from 0 to $-2 \mathrm{~V}$ in 64 possible steps. The D/A converter's output is then inverted and preamplified to a maximum of $+13 \mathrm{~V}$ by an operational amplifier/ buffer. The signal is finally amplified to a maximum of approximately $250 \mathrm{~V}$ by a power amplifier driver circuit and is presented to the elements of the tactile matrix providing a visual display of the pattern being presented. One of the 64 channels is illustrated in Figure 5.

The system requires a total of five voltage sources: $\pm 5 \mathrm{~V}(-5 \mathrm{~V}$ for the $\mathrm{D} / \mathrm{A}$ converters), $\pm 15 \mathrm{~V}$ (for the buffer/preamps), and $+350 \mathrm{~V}$ (for the driver amplifiers). Quiescent current in the high-voltage supply is approximately $2 \mathrm{~mA} /$ channel.

The stimulus waveform presented to the 64 channels may be generated by any external source as long as certain amplitude and offset requirements specific to these D/A converters are met. In this system, we generally employ two sources: an Exact $126 \mathrm{VCF} /$ Sweep function generator and a custom-designed haversine generator. The schematic for the haversine generator is shown in Figure 6, in which the function generator itself is an 8038 integrated circuit that produces sinusoidal, triangular, and square-wave outputs.

The frequency for this particular generator has been fixed at $250 \mathrm{~Hz}$. Although any waveform may be applied to the benders with quite accurate reproduction in the unloaded state, the waveform of even a rectangular pulse reflected by the displacement monitor from a bender contacting the skin with $10 \mathrm{~g}$ of force approximates a haversine. To avoid the possibility of onset and offset transients and to specify better the actual stimulus

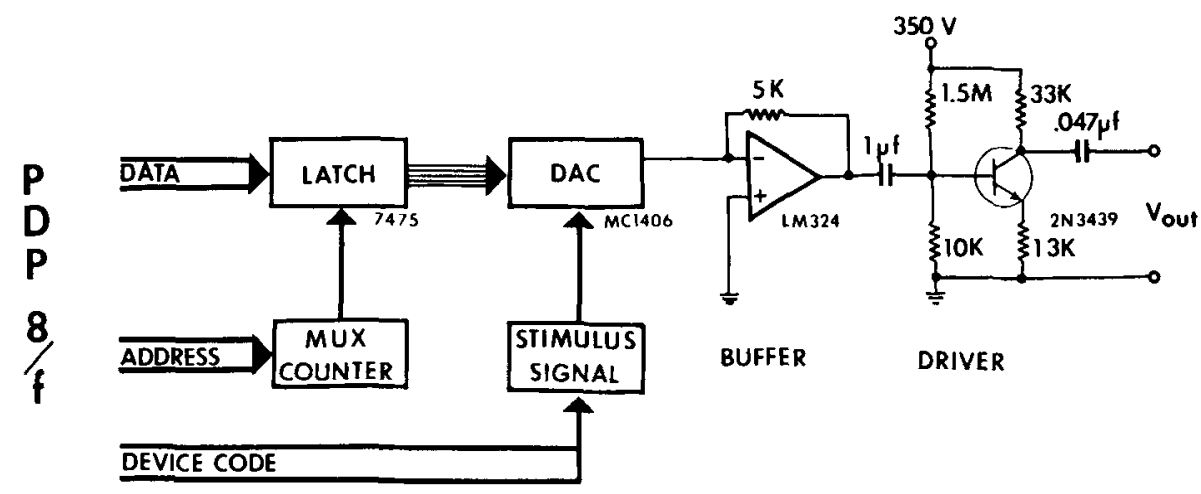

Figure 5. A block diagram of the computer interface system. The matrix controller divides each 12-bit pattern word into 2 bytes, each of which provides the intensity level for one of the $64 \mathrm{D} / \mathrm{A}$ converters. Each bender has its own D/A converter, preamplifier, and driver amplifier. 


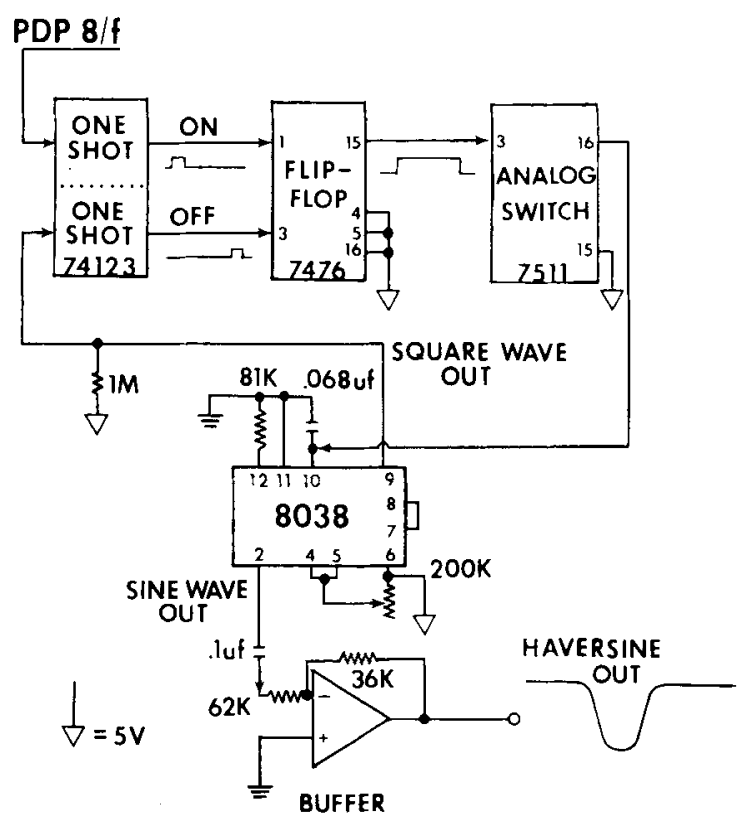

Figure 6. The haversine generator employed in the tactile matrix system to provide the stimulus waveform. Component values shown are for $250-\mathrm{Hz}$ haversines. Owing to the phase relationships between the square-wave and sine-wave outputs of the 8038, the ascending portion of the square wave turns off the generator by tying Pin 10 high through the analog switch, providing a haversine output when the circuit is externally triggered by the computer.

signal, however, we routinely use haversine pulses. Once the 64 channels have been loaded with their individual amplitude information, a trigger pulse or a gating pulse is sent to the appropriate signal generator.

This design allows for truly simultaneous presentation of any number of vibrating points in the display that may be activated at any 1 of 63 levels of intensity independent of each other. Furthermore, the deflections of the active benders are in phase with each other because the signal polarity is identical for each channel and the mounting clamps are oriented in the same direction within the tactile matrix.

\section{COMPUTER SOFTWARE}

As described earlier, spatiotemporal patterns are presented on a frame-by-frame basis, much like that in a movie film. Apparent motion is made possible by slight changes in the position of active elements as the frames are sequentially presented. On the other hand, a "stopframe" capability exists, which we have described as a "static" mode of presentation, in which there are no spatial changes over the period of the stimulus presentation. Other modes, of course, are made possible by appropriate changes in the spatial and temporal parameters specified by the computer pattern software.

The software was written in the PDP-8's assembly language, PAL-8, occupying less than $4 \mathrm{~K}$ of core memory for the program and an additional $12 \mathrm{~K}$ for pattern, command, and response storage. Although the language is specific to this machine, the sequence of events leading up to the production of a pattern on the tactile matrix can illustrate the operation of the system. In the first type of matrix program, patterns are essentially preprogrammed to run in a continuous sequence, with interruption occurring only to collect the observer's responses. In the second type of program, patterns are available for presentation in response to keystrokes from a normal terminal keyboard, in the fashion of a "tactile typewriter." The most recent versions of the matrix programs allow for an interaction between the observer and the patterns presented through the matrix. At the simplest level, the display can imitate a type of "tactile Pong," like the television computer game. Practically speaking, however, there is some suggestion that through such an interaction observers may improve the clarity of their perception of more complex patterns on the skin.

In the first two versions of the software, two levels of pattern programming are necessary. The first stage requires the specification of each frame, or spatiointensitive array, for each instant in time. A frame describes the locations of the vibrators to be activated and the intensity at which each is to be driven. Within a frame, it is possible to present any combination of intensities desired. If the pattern is to move across the tactile array, a series of such frames would be required to describe the changing position of the active elements over time. The second stage in pattern generation involves the specification of the number of times each frame is to be presented (number of repetitions) and the inter- and intraframe intervals in milliseconds. The actual running of the sequence also requires the specification of the sequence of such presentations. The second version of the software, described above, allows for flexibility in defining the sequence of presentations. In this version, although the frames and temporal sequences have been defined by the programmed data, the running of the sequence is determined by predefined keyboard strokes. The latter option is similar in operational style to the Vibratese language, studied by Howell under the Virginia Cutaneous Research Project (Geldard, 1957).

In the most recent modification of the software, two different methods of controlling the presentation of successive frames operate simultaneously. The primary or target pattern has a preprogrammed sequence of movements on the tactile array. The secondary pattern is presented in a sequence that can be controlled by the observer with some type of response device, such as a keyboard or a joystick. The two patterns are either alternated at a controlled rate on the display, or they are presented simultaneously. A trial may consist of an attempt to intercept the target or to maintain some constant relationship between the target's position an the observer-controlled object's position, as in a tracking task. In the first case, a trial would be termi- 
nated by the interception; in the second task, a trial would continue for some predetermined period of time. In both instances, the data collected consist of the target's position, observer's object position, and a measure of the rate of movement of both. The data from all of these procedures are stored on a magnetic disk for off-line analysis using any number of methods. The analyses are usually performed with programs written in BASIC. The most recent version of the interactive program is being written in FORTRAN, with appropriate machine language subroutines.

The software is designed to allow for all time-critical events to be run from core, with program input and data output occurring only before or after the actual trial sequence. By storing the frame data in a manner in which only the active element numbers and their associated intensities are in core, with the reconstruction of the entire 64-byte array required for output occurring during the trial sequence, memory requirements are minimized. Furthermore, efficient use of default levels for the various run-time parameters also minimizes pattern file development time. In most cases, the pattern and command files are, in fact, generated by programs written in the BASIC language, facilitating such manipulations as constructing series of random trials.

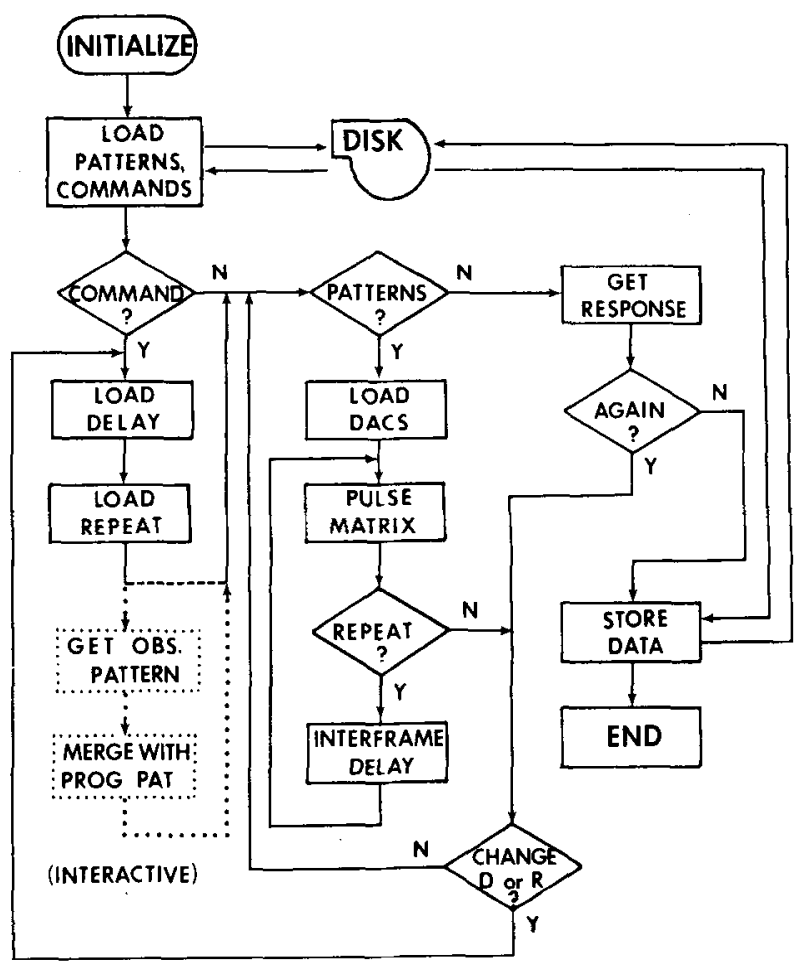

Figure 7. A flowchart of the general form of the matrix software. Processes indicated by the dotted lines are only included in the interactive versions of the program. These allow for a sampling of the observer's response and a modification of the programmed pattern by merging it with the observer's pattern.
The flow diagram for the most general version of the tactile matrix software is shown in Figure 7.

\section{GENERAL DISCUSSION}

The system described was designed for the purpose of examining the pattern-processing capabilities of the skin. To that end, certain assumptions were made about the patterns to be presented that defined particular characteristics of the system. The changing state of the art in electronics, however, and alternative demands for the types of patterns one might desire to present from such a device prompt us to suggest points of departure from this particular design. For example, the frequency response of the amplifier system was optimized for a stimulus waveform frequency of $250 \mathrm{~Hz}$. With a more elaborate design of the amplifier, it would be possible to provide a flatter response over the range of frequencies typically presented in studies of the tactile system. It is notable, however, that from a perceptual standpoint, the vibrotactile loudness (perceived intensity) of a burst of haversine taps that are $1-2 \mathrm{msec}$ in width is relatively immune to changes in the pulse frequency (Rothenberg, Verrillo, Zahorian, Brachman, \& Bolanowski, 1977). From data collected in our laboratory, the tactile thresholds for $400-\mathrm{Hz}$ haversine taps presented at rates of $1-350$ pulses/sec showed only a \pm 5 - $\mathrm{dB}$ shift over the entire range. Furthermore, the perceived intensity of those rates matched to a burst of a $90-\mathrm{Hz}$ sine and $20-\mathrm{dB}$ sensation level varied over only a 6-dB range across nearly 8 octaves of pulse rate. A true sinusoid can be generated by our system if desired, but the amplifier response must be analyzed with the aid of the loaded bender response monitor to insure a flat input to the skin.

A second area for potential improvement is the hardware for specifying the individual intensities of the loci. Advances in technology since the development of our system now provide a single circuit that contains both the data-holding latches and a true four-quadrant multiplying D/A converter. This circuit, the Analog Devices AD7524, was incorporated into the M.I.T. system described earlier, at a considerable savings in circuitry.

Finally, we have received inquiries regarding the possibility of providing different frequencies of stimulation across the surface of the tactile array. Since the signal input to the 64 parallel circuits is common to all, no differential rate is apparently possible. By taking advantage of the relatively rapid changes in frame specification available, however, it is possible to present integral multiples of a given pulse frequency to different sites. This may be accomplished, for example, by deleting the same subset of active elements on alternate presentations, leaving the rest on for each frame. A $2: 1$ ratio of pulse rate thus obtains. Similar interleaving of frames could allow for some coarse control of pulse rate as a function of locus on the array. A more complex 
design would require individual function generators for each locus, presumably programmable, so both intensity and frequency information would have to be specified for each locus. Although complicated in concept, a workable design would include a $\mathrm{D} / \mathrm{A}$ converter for each channel controlling the frequency on a voltage-controlled oscillator, such as the Intersil 8038 integrated-circuit chip used in the haversine generator. If such a design is incorporated, the haversine frequency itself could be varied, thus altering the rise time of the tactile stimulus. As Rothenberg et al. (1977, p. 1006) have pointed out, this is a discriminable dimension, and it may be useful as an additional cue in future complex display systems.

\section{REFERENCE NOTE}

1. Sherrick, C. E., \& Cholewiak, R. W. Matching speech to vision and touch. Paper presented at the Gallaudet Research Conference on Speech Processing Aids for the Deaf, Washington, D.C., May 24-26, 1977.

\section{REFERENCES}

ALLEs, D. S. Information transmission by phantom sensations. IEEE Transactions on Man-Machine Systems, 1970, MMS-11, 85-91.

BuIss, J. C. (Ed.). Tactile displays conference. IEEE Transactions on Man-Machine Systems, 1970, MMS-11, 1-122.

Buiss, J. C. Summary of Optacon-related experiments. In F. A. Geldard (Ed.), Cutaneous communication systems and devices. Austin, Tex: Psychonomic Society, 1974.

Bliss, J. C., Katcher, M. H., Rogers, C. H., \& Shepard, R. P. Optical-to-tactile image conversion for the blind. IEEE Transactions on Man-Machine Systems, 1970, MMS-11, 58-64.

Cholewiak, R. W. Spatial factors in the perceived intensity of vibrotactile patterns. Sensory Processes, 1979, 3, 141-156.

Coulins, C. C. Tactile television-mechanical and electrical image projection. IEEE Transactions on Man-Machine Systems, 1970, MMS-11, 65-71.
Craig, J. C. Pictorial and abstract cutaneous displays. In F. A. Geldard (Ed.), Cutaneous communication systems and devices. Austin, Tex: Psychonomic Society, 1974.

Craic, J. C., \& Sherrick, C. E. Dynamic tactile displays. In W. Schiff \& E. Foulke (Eds.), A sourcebook on haptic perception. Cambridge, England: Cambridge University Press, in press.

Dreyer, D. A., Hollins, M., \& Whitsel, B. L. Factors influencing cutaneous directional sensitivity. Sensory Processes, 1978, 2, 71-79.

Geldard, F. A. Adventures in tactile literacy. American Psychologist, 1957, 12, 115-124.

Geldard, F. A. The human senses (2nd ed.). New York: Wiley, 1972.

Geldard, F. A. (Ed.). Cutaneous communication systems and devices. Austin, Tex: Psychonomic Society, 1974.

Gibson, J. J. Observations on active touch. Psychological Review, $1962,69,477-491$.

Gottheil, E. F., Cholewiak, R. W., \& Sherrick, C. E. The discrimination of vibratory patterns on a tactile matrix. Bulletin of the Psychonomic Society, 1978, 11, 21-24.

Rothenberg, M., Verrillo, R. T., Zahorian, S. A., Brachman, M. L., \& Bolanowski, S. J., JR. Vibrotactile frequency for encoding a speech parameter. Journal of the Acoustical Society, $1977,62,1003-1012$.

RuBin, S. I. A programmable tactile display. Unpublished master's thesis, Massachusetts Institute of Technology, 1978.

NOTE

1. For a $238-\mathrm{Hz} 2-\mathrm{mA}$ peak-to-peak signal driving the bender that is contacting the forearm with $10 \mathrm{~g}$ of force, the resistance of the bender is $500 \mathrm{kohm}$, in shunt with its capacitance, which is .019 microfarads. The complex impedance is $35 \mathrm{kohms}$, and the resulting phase angle is $86 \mathrm{deg}$. For a more detailed description of the electrical characteristics of the 13 x 32 mm Gulton benders, see Rubin (1978).

(Received for publication July 7, 1981; accepted July 28,1981 .) 